\title{
LA INFLUENCIA DE LA INSTITUCIÓN LIBRE DE ENSEÑANZA EN RODOLFO LLOPIS
}

\author{
ADELINA SiRVENT GÁRRIGA \\ Doctora en Sociología
}

\section{Resumen}

Rodolfo Llopis, influido por los principios pedagógicos de la Institución Libre de Enseñanza, intentó renovar la educación: primero como docente en la Escuela Normal de Cuenca, y más tarde como director general de Primera Enseñanza, coincidiendo con el bienio reformista de la Segunda República. Su gran deseo de cambio social le llevó a luchar por la educación y la democracia como pilares fundamentales para la regeneración social, política, pedagógica y cultural. Su formación en la Escuela Superior de Magisterio le puso en contacto con los profesores defensores del ideario de la Institución Libre de Enseñanza, y las influencias de Francisco Giner de los Ríos y Manuel Bartolomé Cossío definieron su perfil de docente y político institucionista.

Palabras clave: Rodolfo Llopis; Institución Libre de Enseñanza; Escuela Superior de Magisterio; Segunda República española; principios educativos.

\begin{abstract}
Rodolfo Llopis, influenced by the pedagogical principles of the Institución Libre de Enseñanza, tried to renew education; first as a teacher in the Normal School of Cuen$\mathrm{ca}$, and later as managing director of Primary Education, coinciding with the reformist biennium of the Second Republic. His great desire for social change led him to fight for education and democracy as fundamental pillars for social, political, educational and cultural regeneration. His training at the School of Education put him in touch with teachers who advocated for the ideology of the Institución Libre de Enseñanza, while the influences of Francisco Giner de los Ríos and Manuel Bartolomé Cossío defined his educational and political profile.
\end{abstract}

Keywords: Rodolfo Llopis; Institución Libre de Enseñanza; College of Education; Second Spanish Republic; educational principles. 


\section{La Escuela Superior de Magisterio}

Rodolfo Llopis ingresó en la Escuela Superior de Magisterio ${ }^{1}$ en 1916; perteneció a la octava promoción. Se presentó al examen de ingreso en la convocatoria de junio y obtuvo plaza para cursar los estudios en la sección de Letras. Finalizó sus estudios en 1919. Ejerció como profesor de Geografía en la Escuela Normal de Cuenca hasta noviembre de 1930, año en que regresó a Madrid para dedicarse de lleno a la política.

La Escuela Superior de Magisterio gozó de un gran prestigio, y a ella acudieron maestros y licenciados interesados en completar su formación pedagógica con las teorías más innovadoras. También Rodolfo Llopis quiso estudiar en este centro, donde conoció las ideas de los institucionistas e hizo grandes amigos entre las figuras más destacadas de la ILE. La influencia que recibió le marcó a lo largo de su vida.

La Escuela contaba con profesores como Ricardo Beltrán y Rózpide, Luis de Hoyos, Domingo Barnés, Luis de Zulueta y Pablo Martínez Strong. Todos tenían una amplia cultura, gran vocación pedagógica y profundos valores humanos, e hicieron de esta Escuela un centro modelo, donde el reducido número de alumnos permitía una enseñanza activa y una estrecha relación entre alumnos y profesores. Estos estaban unidos en su común deseo de responder al espíritu que dio lugar a la creación de la Escuela Superior de Magisterio: elevar el nivel cultural de las Normales $y$, consecuentemente, el de la enseñanza primaria española. Los profesores, influidos por las ideas de la Institución Libre de Enseñanza, quisieron desde el principio poner a sus alumnos en contacto directo con el medio circundante, mediante visitas, excursiones y viajes. Adolfo A. Buylla, director de la Escuela, escribía en 1912: «Respondiendo a aquella necesidad que caracteriza a nuestra Escuela de vivir la enseñanza y de enseñar en vivo, adviértase que muchos [trabajos de los alumnos] se realizan del natural, ya visitando monumentos y museos de arte, bien coleccionando ejemplares de los distintos reinos» (en Ferrer, 1973: 204-205).

1. El Real Decreto de 10 de septiembre de 1911 cambió el nombre de la Escuela, que pasó a llamarse Escuela de Estudios Superiores del Magisterio. Los ministros Amalio Gimeno, Santiago Alba y Francisco Bergamín, a través de sucesivas disposiciones, fueron introduciendo mejoras en la Escuela Superior de Magisterio. Una de ellas era la concesión de becas a todos los alumnos matriculados, y otra que, cuando acabasen sus estudios, saldrían directamente colocados como profesores de Escuela Normal o como inspectores de Enseñanza Primaria. Rodolfo Llopis realizó sus estudios por el Plan de 1914 La octava promoción en la sección de Letras estaba formada por nueve alumnos y siete alumnas. 
En la sección de Letras, la Geografía fue una asignatura importante. Se impartieron las siguientes materias: «Nociones generales de Geografía y Geografía regional», «Metodología de la enseñanza de la Geografía», «Geografía de España», «Geografía universal», «Ampliación de Geografía de España», «Cosmografía y Física del Globo», «Fisiología e Higiene» y «Agricultura», esta última solo para maestros, y para las maestras «Economía doméstica». En 1922, se creó el «Seminario especial de Geografía económica de España $»^{2}$, que estaba dirigido a los alumnos que finalizaban sus estudios en este centro y a los Catedráticos de Geografía de las Normales e Institutos de Enseñanza Media. Ricardo Beltrán y Rózpide organizó este seminario desde su creación hasta 1928. La duración era de tres meses, desde marzo hasta mayo. Se realizaban reuniones semanales «de cotejo y relación de los trabajos realizados de forma individual y colectiva». El objetivo que se perseguía era «organizar y centralizar el estudio sistemático de las regiones españolas, en especial las menos conocidas, desde el punto de vista de la Geografía humana, con especial atención a las cuestiones económicas» (Rodríguez, 1997: 91).

La Escuela Superior de Magisterio formó a profesores que intentaron renovar la enseñanza a través de disciplinas como la Geografía: «Son ellos quienes, a lo largo del tercer y cuarto decenios, estimulan las visitas y excursiones, desarrollan la utilización del mapa, crean museos geográficos e impulsan los estudios locales, las llamadas "monografías de aldea" » (Vilà, 1989: 89-90). Estos profesores difundieron los contenidos, actividades y métodos geográficos en las diferentes Escuelas Normales donde ejercieron: Isidoro Reverte, en las Normales de Albacete y Murcia; Miquel Santaló, en Segovia y Gerona; Leoncio Urabayen, en Pamplona; Pedro Chico, en Soria y Madrid; y Rodolfo Llopis, en Cuenca y Madrid.

Ricardo Beltrán y Rózpide instauró la costumbre de que los alumnos realizaran un trabajo sobre un hecho geográfico: «Un pueblo, una comarca, un género de vida...». Así se iniciaron las monografías geográficas. Estas partían de distintos cuestionarios que indicaban los datos que debían recoger, como el de C. M. Jourdan y el de Albert Demangeon para una investigación regional. Pero, sobre todo, utilizaban el que Luis de Hoyos elaboró para el estudio geográfico de pequeñas localidades y comarcas (Vilà, 1989: 96).

Los trabajos de investigación que desarrollaban los alumnos en los seminarios eran muy importantes, así como las memorias de fin de

2. R. O. de 29 de noviembre de 1922 y R. O. de 20 de abril de 1923.

Anales, 27 (2015), pp. 215-226 
carrera, de planteamiento similar al de las Tesis Doctorales realizadas en la Universidad. La mayoría de los alumnos optaban por monografías geográficas de ámbito local sobre cuestiones metodológicas, clima..., y algunas se dedicaron a la Geografía histórica. Luis de Hoyos Sainz y Ricardo Beltrán y Rózpide dirigieron la memoria de fin de carrera de Rodolfo Llopis, quien eligió un pueblo costero de su provincia natal, Alicante: «Santa Pola. Monografía geográfica» (1919). Estos trabajos fomentaron el interés por la investigación en los alumnos, quienes, más tarde, siendo ya profesores, siguieron ejecutando y promoviendo estudios similares en diferentes lugares de España. Ello supuso la materialización de la investigación sistemática del territorio español, la creación de una Geografía humana de España. Hay que destacar entre los alumnos a Isidoro Reverte, Pedro Chico, Juan Leoncio Urabayen, Miguel Santaló y Rodolfo Llopis, por la labor realizada en diversos trabajos sobre Geografía.

En la Escuela Superior de Magisterio, Rodolfo Llopis entró en contacto con los representantes de la ILE: Luis de Zulueta, su profesor de Pedagogía, y Manuel Bartolomé Cossío. Sobre ellos realizó declaraciones que expresaban su agradecimiento y su influencia. Del primero dijo que preparaba sus clases concienzudamente y que estas se continuaban fuera del aula con inolvidables conversaciones: «Lo recordamos inquietando nuestras conciencias, abriendo nuestros espíritus a un mundo de insospechadas posibilidades. Lo recordamos planteando los más graves problemas morales...» («Nuestros maestros: a Zulueta», en Revista Escuelas Normales, enero de 1928). Y encontramos artículos sobre Manuel Bartolomé Cossío en los que dejó patente su amistad y admiración. En el artículo que dedicó a Fernando de los Ríos en 1946 también habló de aquel y comentó que, cuando terminó el primer curso como profesor de la Escuela Normal de Cuenca, hizo «examen de conciencia. Recapitulé mi actividad pedagógica». Al considerar que había fracasado, decidió visitar a Manuel Bartolomé Cossío para comentárselo (Llopis, 1949): «Y el Sr. Cossío [...] me fue rebatiendo uno a uno todos mis legítimos escrúpulos, animándome, alentándome, convenciéndome de que los frutos de la obra pedagógica no se pueden recoger al día siguiente».

\section{Rodolfo Llopis y los principios pedagógicos de la Institución}

La Institución Libre de Enseñanza tenía entre sus principios educativos esenciales la enseñanza neutral en cuanto a cuestiones políticas y religiosas. Los institucionistas querían «sembrar en la juventud, con la más absoluta libertad, la más austera reserva en la elaboración de sus normas 
de vida y el respeto más religioso para cuantas sinceras convicciones consagra la historia». Rodolfo Llopis, de acuerdo con ese principio fundamental, decía en una intervención parlamentaria en defensa de una enmienda socialista al artículo 48 de la Constitución: "Cueste lo que cueste, hay que respetar la conciencia del niño. [...] [E]ntendemos que la forma de respetar la conciencia del niño es que se quede a la puerta de la escuela toda clase de dogmatismo, lo mismo del tipo religioso...» (20 de octubre de 1931).

La Institución fomentaba en sus alumnos el interés hacia una amplia «cultura general, múltiplemente orientada». Se proponía que los alumnos asimilaran los conocimientos de humanidades que cada época exigía y una educación profesional de acuerdo con sus aptitudes y vocación, «escogida más a conciencia de lo que es uso». Quería prepararlos para que fueran científicos, literatos, médicos...; pero sobre todo quería prepararlos para ser hombres, "personas capaces de concebir un ideal, de gobernar con sustantividad su propia vida y de producirla mediante el armonioso consorcio de todas sus facultades». Rodolfo Llopis escribía al respecto: «La Institución despertaba en sus alumnos el interés hacia la cultura general, siguiendo una orientación educativa que consiste no en aprender las cosas sino en aprender a hacerlas» (1955: 64). Para lograrlo quería que, en el cultivo del cuerpo y del alma, nada les fuese ajeno. Les interesaba el desarrollo intelectual, físico y social, la salud y la higiene, las condiciones estéticas, la formación de la voluntad; y además, «la corrección y nobleza de hábitos y maneras; la amplitud, elevación y delicadeza del sentir; la depuración de los gustos estéticos, la humana tolerancia, la ingenua alegría, el valor sereno, la conciencia del deber, la honrada lealtad, la formación, en suma, de caracteres armónicos dispuestos a vivir como piensan» (1935: 37-38).

Rodolfo Llopis afirmaba que quien pretendiera hacer la historia de la Institución tendría que hacer la historia de España, pues, «aunque ajena a la política militante», por ella desfilaron, en demanda de consejo, desde el maestro rural que quería resolver una dificultad pedagógica «hasta los personajes más encumbrados, a quienes angustiaban difíciles negocios de Estado» (1935: 37). Él, que admiraba profundamente la labor pedagógica de la ILE, había buscado consejo en Manuel Bartolomé Cossío durante su primer curso como profesor, y seguramente lo hizo, aunque no tengamos constancia de ello, en su etapa como Director General.

Siguiendo la estela de la ILE, en la política educativa de la Segunda República se dieron los primeros pasos para que el alumno recibiera una 
educación en valores y se atendieron aspectos educativos que tradicionalmente la escuela pública no contemplaba. En 1931 Rodolfo Llopis envió una circular a los centros en la cual podemos leer: "La supresión de la enseñanza religiosa con carácter obligatorio no debe significar el abandono en la dirección moral de los escolares; por el contrario, [...] el maestro se esforzará, ahora más que nunca, [...] en inspirar en los niños un elevado ideal de conducta» (Circular de la Dirección General de Primera Enseñanza, 13 de mayo de 1931). Ese elevado ideal de conducta se fomentaría, entre otras actividades, con aquellas que la Segunda República creó como complementarias a la escuela: cantinas, colonias escolares y viajes. Respecto a las cantinas, Rodolfo Llopis defendió su función esencial: la educativa. En cuanto a las colonias escolares, el Ministerio de Instrucción Pública organizó una colonia internacional en La Granja, donde niños franceses, ingleses y alemanes convivieron con los niños españoles. En el mismo palacio donde tantas veces se habló de guerras, la República fomentó la conciencia pacifista internacional, al promover actos de esa naturaleza. En los viajes escolares los maestros y los niños hicieron multitud de excursiones por varias provincias españolas. Estas experiencias sirvieron para que unos y otros fueran creando vínculos fraternales al mismo tiempo que enriquecían su cultura: «Queremos que la enseñanza esté fecundada por esos dos principios: el principio del trabajo y el principio de la solidaridad humana» (Llopis, 1933: 227-228).

La educación estética era una disciplina importante, y su aprendizaje se realizaba a través de la contemplación directa de la obra de arte. Con frecuencia, los sábados se veía a alumnos de la Institución recorriendo museos para completar el estudio de esta materia. Visitaban las salas de arte que Madrid les ofrecía a la búsqueda de la lección vivida. Cuando la Historia del Arte estaba empezando a ser disciplina académica en diversos países europeos, la Institución ya la había introducido como elemento de estudio. El Programa institucionista así lo había establecido: «Trabajo intelectual sobrio e intenso, juego corporal al aire libre; larga y frecuente intimidad con la Naturaleza y con el arte» (Programa de la ILE, 1934: 10).

Los principios pedagógicos que la ILE defendió ayudaron a que la Geografía ocupase el lugar que merecía en el sistema educativo. Los institucionistas utilizaron las excursiones escolares como un elemento esencial del proceso de formación. En ellas, fomentaron la observación directa y los conocimientos empíricos que proporcionaban una enseñanza interdisciplinar, intuitiva, continua, activa e integral. Para las clases de Ciencias Naturales y de Ciencias Sociales, también se recurría a la observación 
directa, y los alumnos salían de sus aulas en busca de las fuentes del conocimiento. De las excursiones, nos dice Manuel Bartolomé Cossío que «ofrecen con abundancia los medios más propicios, los más seguros resortes para que el alumno pueda educarse en todas las esferas de su vida» (Cossío, 1966: 25; y Programa de la ILE, 1934: 16-17). Rodolfo Llopis hacía referencia a este principio pedagógico de la Institución diciendo que «no se conformaba con llevar la vida a la escuela; quiere llevar la escuela allí donde la vida se dé»; y añadía que, para valorarla, «hay que situarse en la época [...] y conocer el retraso en que vegetaba la enseñanza española. Solo así se apreciará el sentido revolucionario de su obra» (Llopis, 1955: 65).

Anteriormente se ha citado un artículo en el que Rodolfo Llopis describía las actividades que realizaba con sus alumnos fuera del aula, en el que podemos comprobar su clara apuesta por la metodología institucionista. Afirmaba allí que la única influencia eficaz con los alumnos se consigue estando mucho tiempo fuera de la clase, charlando con ellos, realizando salidas, excursiones y viajes. Este principio pedagógico lo asumió la educación de la Segunda República, y un ejemplo de ello lo encontramos en la circular de la Dirección General de Primera Enseñanza de 12 de enero de 1932, en la que podemos leer: «El maestro utilizará todos los grandes valores educativos que encierra el ambiente geográfico. La fábrica, el taller, la granja, el mar, todo lo que constituya la fisonomía económica y espiritual de aquella zona ha de ser familiar al niño y a la escuela» (1).

Respecto a los exámenes y a la disciplina de los alumnos, podemos ver los principios de la Institución: «absoluta protesta, en cuanto a disciplina moral y vigilancia, contra el sistema corruptor de exámenes, de emulación de premios y castigos, de espionaje y de toda clase de garantías exteriores» (Llopis, 1935: 37-38). Para ser discípulo de la ILE el alumno debía aceptar su proyecto educativo y desear ser educado. El único premio era realizar bien la tarea educativa; el único castigo no realizarla, y por lo tanto, no mantener el nivel que la comunidad educativa exigía. Para evaluar los conocimientos adquiridos, Francisco Giner de los Ríos proponía utilizar el cuaderno del alumno donde se anotaba el trabajo realizado a lo largo de la escolaridad y comprobar así los progresos alcanzados. La supresión de exámenes junto con la eliminación de toda fórmula externa de disciplina eran dos de los aspectos originales de la Institución. La idea del cuaderno que proponía Francisco Giner de los Ríos aparece en un artículo de Rodolfo Llopis: «El Diario de clase». Para él, el Diario era «un fuerte motivo de honda colaboración entre profesores y alumnos. Es una manera 
de incorporarlos a las inquietudes de la clase» (1924: 8). Los alumnos tenían que valorar y calificar el trabajo de sus compañeros, como manifestación de su llamamiento constante al sentimiento de la responsabilidad.

La coeducación era otro principio esencial del régimen escolar de la Institución, ya que no había razón para prohibir en la escuela lo que de forma natural se daba en la sociedad. Se consideraba como uno de los «resortes fundamentales para la formación del carácter moral y la pureza de costumbres»; además, «es el más poderoso para terminar con la situación actual de inferioridad de la mujer, inferioridad que no empezará a desaparecer hasta que aquella se eduque, en cuanto a la cultura general, no solo "como", sino "con" el hombre» (Llopis, 1935: 38). La implantación de la coeducación generó en la sociedad española una gran polémica. Desde el Ministerio de Instrucción Pública se propuso una implantación progresiva. El 28 de agosto de 1931 se estableció la enseñanza mixta en dos Institutos de enseñanza media en Madrid y en Barcelona. En septiembre se estableció la coeducación en las Escuelas Normales, y el 17 de abril de 1932 en las escuelas anejas de las Normales ${ }^{3}$. Sabemos que Rodolfo Llopis estaba de acuerdo con la coeducación, y así lo expresó: «Razones pedagógicas, sociales y políticas aconsejan establecerla. Y se ha establecido sin dificultad ni tropiezo alguno. A pesar de las campañas de la prensa reaccionaria» (1935: 38).

Se utilizó la metodología activa de Johann Heinrich Pestalozzi y Friedrich Froebel, que la Institución aplicó a todos los grados. En todos ellos cabía «la intuición, trabajo personal y creador, procedimiento socrático, método heurístico, animadores y gratos estímulos, individualidad de la acción educadora en el orden intelectual como en todos» (Programa de la ILE, 1934: 12). Rodolfo Llopis suscribía los principios pedagógicos de la Institución; así lo manifestó en repetidas ocasiones: en la circular de la Dirección General de Primera Enseñanza de 12 de enero de 1932; también durante su actividad docente en la Escuela Normal de Cuenca, y, por último, al conocer la Escuela Nueva y el método de Ovide Decroly durante el curso escolar en que estuvo en Suiza, Bélgica y Francia para ampliar su formación.

Según establecía el Programa, en la Institución no existía separación entre la escuela de párvulos, la primaria y la secundaria: «los tres periodos

3. La enseñanza mixta en los Institutos se implantó por Decreto de 28 de agosto de 1931 (Gaceta del 29); en las Escuelas Normales, por Decreto de 29 de septiembre de 1931 (Gaceta del 30); en las Escuelas Anejas de las Normales, por Orden de 17 de abril de 1932. 
constituyen uno solo y continuo: el de la educación general». El alumnado estaba dividido en secciones, según el grado de desarrollo, y todos debían aprender las mismas disciplinas en las primeras secciones que en las últimas, siempre de acuerdo con su nivel madurativo. Las lenguas clásicas eran las únicas materias que la Institución creía que no debían imponerse a todos los niños, sino solo a aquellos que mostrasen un especial interés. Estudiaban desde el primer grado todas las materias que constituían la base de la cultura general de la época: «la lengua materna y las vivas, las ciencias naturales, matemáticas, física, química, sociales, filosofía, la historia de la civilización, la geografía, la literatura, la teoría y la historia del arte, el dibujo y el modelado, la música y el canto, el trabajo manual...». Rodolfo Llopis defendió ante la Cámara el mismo planteamiento de la ILE para la organización de las diferentes etapas del sistema educativo. Y, en la citada defensa de la enmienda socialista al artículo 48 de la Constitución, dijo: «Hay que conseguir que, desde la escuela maternal hasta la Universidad, todas las instituciones que existan en el país [...] estén todas ellas íntimamente coordenadas y enlazadas, como si fuesen los eslabones de una misma cadena» (20 de octubre de 1931).

La Institución deseaba que sus alumnos pudieran utilizar pronto y ampliamente los libros como fuente capital de cultura, pero no empleaba los libros de texto, ni se recurría a la memorización de las lecciones. Pensaba que todo ello contribuía a mecanizar el trabajo de clase. Proponía como funciones del maestro las de despertar y mantener vivo el interés del niño, excitar su pensamiento, sugerirle cuestiones y nuevos puntos de vista, enseñarle a razonar con rigor y a resumir con claridad y precisión los resultados. Para desarrollar la memoria y enriquecerla, se seleccionaba fragmentos de textos literarios que los niños aprendían. En la citada circular enviada por Rodolfo Llopis a los centros, una vez aprobada la Constitución, leemos: «La escuela libresca de ayer ha de ser superada por la escuela activa de hoy. Los horarios viejos y los programas rutinarios han de ser superados por los centros vivos de interés y por la libre curiosidad del niño» (12 de enero de 1932). El decreto de 7 de agosto de 1931 ordenaba la creación de bibliotecas en todas las escuelas públicas. El Patronato de Misiones Pedagógicas era el encargado de la adquisición, selección y distribución de los libros. El fondo de las bibliotecas quedó integrado por obras destinadas tanto para el disfrute de los niños como de los adultos, a los que también iban dirigidas (Patronato de Misiones Pedagógicas, 1933: 64). Esta medida tomada por el Ministerio de Instrucción Pública demostraba, una vez más, el deseo de culturizar a la población. 
La Institución consideraba indispensable la activa colaboración de las familias para que su obra fuera eficaz. El niño debía vivir en familia y a ella debía volver todos los días, cuando finalizara la escuela. Esta era para el niño lo que la esfera profesional y las complejas relaciones sociales para el hombre adulto, y en ninguno de ambos casos había motivo para que se suprimiera la vida familiar, pues nada entendían tan nocivo para la educación del niño como el manifiesto o latente desacuerdo entre su familia y la escuela. "Nada, por el contrario, tan favorable como el natural y recíproco influjo de una en otra» (Programa de la ILE, 1934: 18). Para Antonio Jiménez-Landi la Institución no solo se adelantó a la Escuela Nueva, sino que la superó, ya que mientras esta impuso el internado, en la Institución se defendía que el niño debía vivir en familia completando la acción educativa de la escuela en el hogar. Es importante destacar la convivencia que establecieron maestros y discípulos, la cual se prolongaba en las excursiones y en las colonias de verano (1976: 48-54). En cuanto a la relación familia-escuela, a Rodolfo Llopis le preocupaba el absentismo escolar que se daba en España en 1931. Por ello, ante las Cortes habló de la necesidad de dar «subsidios familiares» para que los niños no dejaran de asistir a la escuela y ayudar económicamente a sus padres. Él sabía que la escuela era la que debía acercarse a las familias: «la escuela establecerá esa relación íntima con la vida del trabajo y con la vida del hogar, donde tanta influencia puede ejercer. La escuela procurará interesar a los padres y a las madres organizando enseñanzas que respondan a sus inquietudes, organizando bibliotecas, lecturas, audiciones y conferencias» (Circular, 12 de enero de 1932).

La política educativa del Ministerio de Instrucción Pública asumió la mayoría de los principios educativos de la ILE, y a ello hizo referencia Rodolfo Llopis cuando escribió que la República no olvidó la deuda que tenía con Francisco Giner de los Ríos. Según Llopis, si la República había sido posible era gracias a la madurez política y a la conciencia cívica del pueblo español. A ello llegaron los españoles gracias a dos influencias: una intelectual y otra obrera. La primera se debía directamente a Francisco Giner de los Ríos; la segunda, a Pablo Iglesias. Aquel supo despertar y crear un ideal para la vida; este, además, supo despertar la necesidad de ofrecer la vida al servicio del ideal: «La República, agradecida a estos dos hombres, al votarse la Constitución, regaló la construcción de dos magníficos grupos escolares: uno, a la ciudad de Ronda, cuna de Giner de los Ríos; otro, a la ciudad de El Ferrol, cuna de Pablo Iglesias. A mí me cupo el honor de redactar el decreto-ofrenda» (Llopis, 1955: 67). Al instalarse 
en el despacho de la Dirección General de Primera Enseñanza, había colocado en el sitio de honor un retrato de Pablo Iglesias, y a su lado los de don Francisco Giner de los Ríos y don Manuel Bartolomé Cossío. Llopis se complacía en explicar a las visitas que entraban en el despacho y miraban los retratos con mal disimulada sorpresa lo que significaba aquel modesto homenaje que rendía «a los tres grandes educadores que tanto habían contribuido a forjar la conciencia revolucionaria del país» (1933: 21).

Con motivo del cincuentenario de la ILE, Llopis escribió un artículo en La Lucha donde afirmaba: «La Institución ha producido una intensa renovación espiritual en todos los órdenes de la vida nacional» (1926: 1). Decía que el día que se pudiera hacer inventario de todo lo que la Institución y Francisco Giner de los Ríos habían dado a España, los españoles se asombrarían, pues ambos acogieron a cuantos se les acercaron, desde el maestro de escuela al político encumbrado que planteaba altos problemas de Estado.

Sin duda, la República se encontró con muchos docentes formados en los principios pedagógicos de la Institución, pero también con otros que, conocedores de sus preceptos, los asumieron por propia iniciativa y pusieron en práctica en sus aulas. Efectivamente la Institución había ejercido una gran influencia en la educación española, y esta continuó intensificándose durante los primeros años de la República. Los que vivían en provincias podían comprobar que, cuando llegaba «un profesor o un maestro liberal, con ideas claras acerca de los problemas vitales, y con suficiente decisión espiritual para no humillar su conciencia de hombre», el comentario habitual era: «Ese es de la Institución...», aunque nunca hubiera tenido contacto con ella ni con sus hombres. Y es que, «como en el ambiente español flota la influencia "institucionista", no tiene nada de particular que hasta él "arribara la inteligencia amorosa" » (Llopis, 1926: 1).

\section{Bibliografía citada}

Cossío, M. B., De su jornada, Madrid, Aguilar, 1966.

Ferrer C. Maura, S., La Escuela de Estudios Superiores del Magisterio (1909-1932), Madrid, Cedesa, 1973.

HERAS J. DE LAS, «Rodolfo Llopis, añoranza de Cuenca», Elbanzo [Cuenca], 6 (1976).

JiMÉNEZ-LANDI, A., «La ILE en sus coordinadas pedagógicas», Revista de Educación, 243 (1976), pp. 48-54. 
LLOPIS, R., «El Diario de clase», Revista de Escuelas Normales [Guadalajara], 11 (1924).

— «Este es un libro de paz», La Lucha [Cuenca], 14 de noviembre de 1926.

- «Nuestros maestros: a Zulueta», Revista Escuelas Normales (enero de 1928).

- Circular de la Dirección General de Primera Enseñanza, 13 de mayo de 1931.

- Discurso pronunciado para la defensa de una enmienda al artículo 48 de la Constitución, 20 de octubre de 1931.

- Circular de la Dirección General de Primera Enseñanza, 12 de enero de 1932.

- La revolución en la escuela, Madrid, Aguilar, 1933.

— «El señor Cossío: una vida luminosa que se apaga», Leviatán, 17 (1935).

- «Nuestro don Fernando en Instrucción Pública», Le Socialiste [Toulouse], 9 de junio de 1949.

- «Francisco Giner de los Ríos y la reforma del hombre», Cuadernos del Congreso por la Libertad de la Cultura [París], 4 de marzo de 1955, pp. 60-67.

MoratinOS IGLESIAS, J., El pensamiento pedagógico del alicantino Rodolfo Tomás y Samper, Alicante, Ayala, 1988.

Patronato de Misiones Pedagógicas, Madrid, Ministerio de Instrucción Pública y Bellas Artes, 1933.

Programa de la Institución Libre de Enseñanza, Madrid, 1934.

Rodríguez Esteban, J. A., «La Geografía en la Escuela Superior de Magisterio (1909-1932)», Ería, 42 (1997), pp. 89-106.

Vilà Valentí, J., El conocimiento geográfico de España, Madrid, Síntesis, 1989.

Fecha de recepción: 24-7-2015

Fecha de aceptación: 8-12-2015 